\title{
Radiation Oncologists' View on the Zurich Consensus
}

\author{
Rainer Souchon ${ }^{\mathrm{a}} \quad$ Marie-Luise Sautter-Bihl ${ }^{\mathrm{b}}$ Felix Sedlmayer ${ }^{\mathrm{c}}$ Frederik Wenz $^{\mathrm{d}}$ \\ Wilfried Budach ${ }^{\mathrm{R}}$ Rolf Sauer ${ }^{f}$ on behalf of the Breast Cancer Expert Panel \\ of the German Society of Radiation Oncology (DEGRO)
}

aKlinik für Radioonkologie, Universitätsklinikum Tübingen,

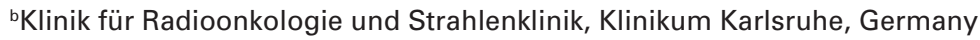

cUniversitätsklinik für Radiotherapie und Radio-Onkologie, Universitätsklinikum der Paracelsus Medizinischen Privatuniversität, LKH,

Salzburg, Austria

dKlinik für Strahlentherapie und Radioonkologie, Universitätsmedizin Mannheim,

eKlinik für Strahlentherapie und Radiologische Onkologie, Universitätsklinikum Düsseldorf,

'Strahlenklinik, Universitätsklinikum Erlangen, Germany

\section{Keywords}

St. Gallen Consensus · Radiation Oncology ·

\section{Breast Cancer}

\section{Schlüsselwörter}

St. Gallen-Konsensus · Radioonkologie · Mammakarzinom

\author{
On: Untch M, Gerber B, Harbeck N, et al. 13th \\ St Gallen International Breast Cancer Conference \\ 2013: Primary Therapy of Early Breast Cancer \\ Evidence, Controversies, Consensus - Opinion \\ of a German Team of Experts (Zurich 2013). \\ Breast Care 2013;8:221-229
}

\section{Dear Colleagues,}

Given the heading 'Consensus' in the June issue of Breast Care 2013, the opinion of a German team of experts regarding recommendations and statements of this year's 13th St. Gallen International Breast Cancer Conference 2013: Primary Therapy of Early Breast Cancer has been published [1].

Without questioning the relevance of the St. Gallen Consensus Conference [1], we would like to point out that the conference recommendations reflect expert opinions based on majority votes of panelists from 19 countries, who represent various fields of expertise in substantially differing health care systems. Commendably, the German team of experts provided additional comments on the recommendations of the 2013 Conference, allowing these international statements to be related to common practice in Germany. While recognizing the expertise in breast cancer treatment of the German team, it should be stressed that none of the authors is a radiation oncologist. This in fact applies to the great majority of the St. Gallen Conference panelists. We, therefore, feel motivated to complement the consensus of the conference and the statements by the German team by highlighting the perspective of radiation oncology on selected issues of the consensus with which we disagree.

\section{Subtopic: Radiation after Breast-Conserving Surgery}

The German expert team agreed with a $68 \%$ majority of the panelists that patients with breast-conserving surgery do not necessarily have to receive adjuvant radiotherapy (RT). It was stated that the omission of irradiation may be justifiable in elderly low-risk cancer patients and in cases with co-morbidity with limited life expectation $(<10$ years).

Comment: No subgroup of patients has yet been identified for whom RT can be safely omitted. According to the standard of care and good clinical practice, we agree that it may be reasonable to refrain from $\mathrm{RT}$ in frail patients with comorbidities substantially impairing life expectation [2, 3-6]. However, any decision should ultimately be made by the patient, respecting individual preference. The level of evidence (LoE 1) regarding the subgroup of 'low risk' and/or elderly patients demonstrates that RT provides a significant benefit in terms of local tumor control for this cohort ( $9 \%$ vs. $2 \%$ ) [2-7].

\section{Subtopic: Hypofractionated (Short Course) RT}

Comment: A total dose of $45 \mathrm{~Gy}$, administered in 15 fractions, is in no way a standard option: The LoE 1 was obtained in the START B trial with a single dose of 2.66 Gy in 15 fractions or 42.5 Gy in 16 fractions in Canadian trials [8-10].

Hypofractionated schedules in combination with sequential and synchronous boost (limited to the tumor region) might be an option, and are currently being evaluated in prospective clinical trials, but are not recommended outside clinical trials due to the lack of mature data from randomized controlled trials [11].

\section{KARGER}

Fax +497614520714

Information@Karger.com

www.karger.com
(C) 2013 S. Karger GmbH, Freiburg

$1661-3791 / 13 / 0086-0448 \$ 38.00 / 0$

Accessible online at:

www.karger.com/brc
Prof. Dr. med. Rainer Souchon

Dept of Radiation Oncology

University Hospital Tuebingen

Hoppe-Seyler-Str. 6

72076 Tuebingen, Germany

Rainer.Souchon@med.uni-tuebingen.de 


\section{Subtopic: Postmastectomy Radiation Therapy (PMRT)}

of the Thoracic Wall and Regional Lymph Nodes

The conference consensus was that in cases with 1-3 positive lymph nodes PMRT should not be routinely indicated unless additional risk factors are present. The panelists further voted for PMRT in patients with positive sentinel lymph nodes (SNs) who receive no axilla dissection (AD). The German expert team disagreed with this vote. The St. Gallen panelists stated that PMRT is not necessarily indicated in pN0 patients with G3, triple-negative breast cancer, vascular invasion and/or Her2 positivity (even in young age). Although the German expert team agreed with this, they suggested the inclusion of additional risk factors.

Comments: Summarizing the data from the Early Breast Cancer Trialist's Collaborative Group, presented in 2009 [7], clear benefit has been confirmed when PMRT is administered to the subgroup of patients with 1-3 positive lymph nodes.

We agree to the vote of the panelists that PMRT is indicated if no $\mathrm{AD}$ is performed in patients with a positive $\mathrm{SN}$. We understand the caution of the German expert teams regarding the omission of AD in cases with positive SN. However, the first results of the AMAROS trial [12] indicate a similar effectiveness of axillary RT compared to AD (5-year recurrence rate $1.03 \%$ vs. $0.54 \%$, not significant) with significant lower incidence of lymphedema after RT. Of note, only $17.8 \%$ of the patients had mastectomy, and a subgroup analysis has not yet been presented.
Considering pN0 patients with G3, triple-negative breast cancer, vascular invasion and/or Her2 positivity, in a systematic review by Rowell [13], several tumor biological features such as vascular invasion, G3 grading, tumor size and especially premenopausal status have been identified to characterize an 'intermediate' recurrence risk. The probability of relapse with more than 1 of these factors amounted to $\geq 15 \%$ [13]. These data support considering PMRT in these cases - as recommended in various national guidelines $(\mathrm{NCCN}$, French Group, NZGG and Belgian KCE) [14].

\section{Subtopic: Irradiation of the Nodal Areas}

The panelists and the German expert team agreed that adjuvant irradiation of the nodal areas should not be based on the response to neoadjuvant chemotherapy (NACT), and that adjuvant irradiation of the nodal areas is not automatically indicated. A majority of the panelists also did not agree with RT of the periclavicular lymphatics or the ipsilateral mammaria interna node area for cases in which nodal irradiation was indicated.

Comment: We were unable to identify evidence in the relevant literature that supports this recommendation. In contrast to the above statement, the German expert team recommended $\mathrm{RT}$ of the regional lymphatics in patients with $\mathrm{pN} 2 \mathrm{a},(\mathrm{p}) \mathrm{N} 3 \mathrm{a}-\mathrm{c}$ and individually in $\mathrm{pN} 1 \mathrm{a}$ breast cancer patients [1].

\section{References}

1 Untch M, Gerber B, Harbeck N, et al.: 13th St Gallen International Breast Cancer Conference 2013: primary therapy of early breast cancer evidence, controversies, consensus - opinion of a german team of experts (Zurich 2013). Breast Care 2013; 8:221-229.

2 www.ago-online.de.

3 Hughes KS, Schnaper LA, Bellon JR, et al.: Lumpectomy plus Tamoxifen with or without irradiation in women age 70 years or older with early breast cancer: Long-term follow-up of CALGB 9343. J Clin Oncol 2013;31:2382-2387.

4 Sautter-Bihl ML, Sedlmayer F, Budach W, et al.: When are breast cancer patients old enough for the quitclaim of local control? Strahlenther Onkol 2012;188:1069-1073.

5 Cohen RJ, Li L, Citron W, Oh M et al.: Improved survival with adjuvant radiation in elderly women with early-stage breast cancer. Int J Radiat Oncol Biol Phys 2012;84 Suppl., S33/34, abstract 82.
6 Biganzoli L, Wildiers H, Oakman C, et al.: Management of elderly patients with breast cancer: Updated recommendations of the International Society of Geriatric Oncology (SIOG) and European Society of Breast Cancer Specialists (EUSOMA). Lancet Oncol 2012;13:e148-160.

7 Darby S, on behalf of the Early Breast Cancer Trialists' Collaborative Group University of Oxford, GB: Overview of the randomised trials of radiotherapy in early breast cancer. SABCS 2009 [MS3-1].

8 Whelan TJ, Pignol JP, Levine MN, et al.: Longterm results of hypofractionated radiation therapy for breast cancer. N Engl J Med 2010;362:513-520.

9 Haviland JS, Agrawal R, Aird E, et al.: on behalf of the START Trialists. The UK START (Standardisation of Breast Radiotherapy) Trials: 10-Year follow-up results. SABCS 2012; S4-1.

10 Herbert C, Nichol A, Olivotto I, et al.: The impact of hypofractionated whole breast radiotherapy on local relapse in patients with grade 3 early breast cancer: A population-based cohort study. Int J Radiat Oncol Biol Phys 2012;82:2086-2092.
1 Sedlmayer F, Sautter-Bihl ML, Budach W, et al.: Breast Cancer Expert Panel of the German Society of Radiation Oncology (DEGRO). Is the simultaneously integrated boost (SIB) technique for early breast cancer ready to be adopted for routine adjuvant radiotherapy? Statement of the German and the Austrian Societies of Radiooncology (DEGRO/ ÖGRO). Strahlenther Onkol 2013;189:193-196.

12 Rutgers ETJ, Donker M, Straver ME, et al.: Radiotherapy or surgery of the axilla after a positive sentinel node in breast cancer patients: Final analysis of the EORTC AMAROS trial (10981/22023). J Clin Oncol 2013;31(Suppl; abstr LBA1001).

13 Rowell NP: Radiotherapy to the chest wall following mastectomy for node-negative breast cancer: A systematic review. Radiother Oncol 2009;91:2332.

14 Abi-Raad R, Boutrus R, Wang R, et al.: Patterns and risk factors of locoregional recurrence in T1-T2 node negative breast cancer patients treated with mastectomy: Implications for postmastectomy radiotherapy. Int J Radiat Oncol Biol Phys 2011; 81:e151-157. 


\section{Author's Reply}

We would like to thank Prof. Rainer Souchon and his colleagues for providing us with their valuable comments regarding our article '13th St. Gallen International Breast Cancer Conference 2013: Primary Therapy of Early Breast Cancer Evidence, Controversies, Consensus - Opinion of a German Team of Experts (Zurich 2013)'.

First of all, let us summarize the composition of the German writing committee, which is comprised of 5 directors of large multidisciplinary breast cancer centers in Germany (M.U., B.G., N.H., C.J., V.M.), who also represent major German breast cancer study groups (AGO-B, WSG), 1 medical oncologist, and 2 representatives from the German Breast Group (G.v.M., S.L.). 2 members of the writing committee (M.U., S.L.) are also members of the current St. Gallen panel.

The panel of the St. Gallen Consensus Conference had 50 experts from 19 countries. The Consensus was published in August 2013 [1]. In the International Consensus Committee from St. Gallen, all disciplines involved in the diagnosis and treatment of breast cancer patients were represented; the panel included Prof. J. R. Harris from the Department of Radiation Oncology at the Dana-Farber Cancer Institute Boston (USA), Prof. Jacek Jassem, Department of Oncology and Radiotherapy from the M. University of Gdansk (Poland), and Prof. F. Sedlmayer, Department of Radiotherapy and Radiation Oncology, Medical University Salzburg (Austria).

The German Expert Opinion Conference, held immediately after the meeting in St. Gallen, aimed at commenting on the St. Gallen Consensus on the one hand, and at harmonizing the St. Gallen Consensus with the National German Guidelines (AGO and S3 Guidelines) and international guidelines based on evidence-based principles.

Here are our answers in detail.

\section{Subtopic: Radiation after Breast-Conserving Surgery}

The original wording of the St. Gallen paper reads: 'A large majority of panel members thought that there were definable groups of patients not requiring radiotherapy following a breast-conserving surgery, and that these might include the elderly and those with substantial co-morbidity'. As always, therapeutic decisions should be made by the patient in knowledge of risks, side effects, and benefits. The same applies for the decision for or against chemotherapy and immunotherapy.

\section{Subtopic: Hypofractionated (Short Course) RT}

The original wording of the St. Gallen paper is 'The Panel strongly agreed that 'short course radiotherapy', such as $40 \mathrm{~Gy}$ in 15 or 42.5 Gy in 16 fractions, could be offered as a standard for at least some patients, with a slim majority think- ing that this would be suitable for almost all patients. The Panel agreed that short course radiotherapy was an option whether or not a boost to the tumor bed was planned'.

We would like to draw the attention to the fact that the 'U.K. standardization of breast radiotherapy (START) trials of radiotherapy, hypofractionation for treatment of early breast cancer: 10 follow up results of 2 randomized controlled trials' has just been published in Lancet Oncology [2]. In START A, 2,236 women were randomized to 50 Gy (25 fractions over 5 weeks) compared to 41.6 Gy or 39 Gy in 13 fractions over 5 weeks. In START B, 2,215 women were randomized to 50 Gy (25 fractions over 5 weeks) compared to $40 \mathrm{~Gy}$ in 15 fractions over 3 weeks. The median follow-up of START A was 9.3 years and of START B 9.9 years. Patients were recruited after complete excision of primary invasive cancer (pT1-3a, pN0-1, M0). The interpretation of these data by the authors in Lancet Oncology is the following: 'Longterm follow-up confirms that appropriately dosed hypofractionated radiotherapy is safe and effective for patients with early breast cancer. The results support the continued use of 40 Gy in 15 fractions, which has already been adopted by most U.K. centers as a standard for women requiring adjuvant radiotherapy for invasive early breast cancer'.

In the Editorial accompanying the article by Bruce Haffty and Thomas Buchholz from the Department of Radiation Oncology, New Jersey Medical School and the Division of Radiation Oncology at the University of Texas, M.D. Anderson, the conclusion is the following: 'Overall, the results of the START trials have significant clinical implications for patients as the hypofractionated regimen might provide many patients with a more convenient treatment. CHOOSING WISELY is a national campaign in the USA aiming at promoting conversations between physicians and patients about the overuse of tests and procedures and effective treatment choices. The American Society of Radiation Oncology has encouraged CHOOSING WISELY to promote discussion of hypofractionation for appropriately selected patients with breast cancer. Widespread use of a 3-week course of radiation would reduce health-care costs, whilst maintaining high treatment quality without compromising outcome. The data from the START trials presented here clearly support this position.' [3].

We agree with the authors of the Letter to the Editor that combination of hypofractionated schedules with sequential and synchronous boost (limited to the tumor region) is an option, which is currently being evaluated in prospective clinical trials in Germany and Austria. We therefore encourage all breast cancer centers to include their patients into trials such as the one chaired by Prof. Sedlmayer and colleagues from Salzburg, including the dedicated breast cancer centers in Germany that have the possibility to use intraoperative radiotherapy (IORT). 


\section{Subtopic: Post-Mastectomy Radiation Therapy (PMRT)}

of the Thoracic Wall and Lymph Nodes

The original wording of the published St. Gallen Consensus paper is: 'Post-mastectomy radiotherapy was considered indicated by almost all panel members for patients with 4 or more positive nodes, while the majority would not advise post-mastectomy irradiation for those with 1-3 positive nodes, except in the presence of adverse tumor pathology.

The panel was content to omit post-mastectomy radiotherapy for pathologically not involved nodes even when fewer than 8 nodes had been examined and if the tumor is less or equal to $5 \mathrm{~cm}$. Two-thirds felt that radiation therapy should be given after mastectomy if the sentinel node was positive, when not followed by axillary dissection.'

The reason why the German expert team disagreed with this vote was the lack of data. In the ACOSOG trial Z0011 published by Giuliano et al. [4], patients with 1-2 involved sentinel lymph nodes (SNs) were eligible only if breast-conserving surgery followed by sequential field irradiation of the ipsilateral breast was performed. In this study, patients with mastectomy were excluded and, therefore, in cases with 1-2 involved SNs, axillary node dissection (AD) is still mandatory if a mastectomy has to be performed.

The AMAROS trial shows that the outcome of the patients is the same if - in case of involved SNs - AD or a regional RT including the axillary region is performed. The study has major limitations and does not support the use of axillary RT for patients who have involved SNs and receive a mastectomy. According to the ACOSOG trial, there is no need for additional RT on the axilla in patients who received no AD even if they had 1-2 involved axillary SNs. The authors of the Letter to the Editors focused on the weakness of the AMAROS trial to answer the question of whether an additional RT of regional lymph nodes is needed in the case of patients with mastectomy and involved SNs: only $17.8 \%$ of the patients had a mastectomy, and therefore, a subgroup analysis has not been performed.

The original statement of the St. Gallen Consensus paper for patients with uninvolved axillary lymph nodes is: 'However, the panel strongly rejected needing radiotherapy solely based on grade 3 lymphovascular invasion, HER2-positive status or triple-negative disease.'

The authors of the Letter to the Editor quote various national guidelines like NCCN, French group, NZGG, and Belgian KCE. On the other hand, one could also quote other guidelines such as the German AGO and S3 in which post-mastectomy RT is not generally recommended in patients with node-negative status. One of the radiation oncologists who is a co-author of the German Consensus Guidelines (S3 and AGO) is also the first author of the Letter to the Editors (Rainer Souchon).

\section{Subtopic 'Irradiation of the Nodal Areas'}

The original consensus wording is as follows: 'Areas to be radiated following mastectomy and axillary dissection should not be influenced by any neoadjuvant systemic therapy or by the intrinsic subtypes of the tumor. There was no clear agreement about the necessity to include the subclavicular fossa, though trials have routinely included this area. Most panel members would not include the internal mammary chain and a strong majority felt that the axilla should not be irradiated after dissection'.

Our comment to this point is the following: With reference to the AGO Guidelines [5] in which the first author of the comment has been a member for many years, the German experts recommend supra-infra clavicular field (SICF) irradiation for patients with $\mathrm{pN} 2 \mathrm{a}$ and $\mathrm{pN} 3 \mathrm{a}-\mathrm{c}$ tumors. In addition, SICF irradiation is only recommended in individual cases in stage pN1a if level III lymph nodes are affected or if axillary surgery cannot reach $\mathrm{R} 0$ margins.

In general, the recommendation of regional lymph node irradiation comes from studies published in the 1990s. In the meantime, systemic treatment approaches have changed dramatically: routine use of anthracyclines and taxanes as adjuvant chemotherapy; routine use of trastuzumab in patients with HER2-overexpressing disease; as well as routine use of endocrine treatment in patients with hormone receptor-positive tumors, premenopausal patients and patients with uninvolved axillary lymph nodes, which was not generally recommended until the end of the 1990s.

In addition, especially in Germany, many patients have been included in neoadjuvant trials, and an increasing rate of high pathological complete responses has been seen, even in patients who had involved axillary lymph nodes before neoadjuvant therapy. Therefore, we, together with our colleagues from radiation oncology, welcome studies in which irradiation of regional nodes is omitted if the patient has a pathological complete response after neoadjuvant therapy. The integration of intraoperative RT is increasing in Germany, and one of the authors of this comment (Prof. Sedlmayer from Salzburg) chaired a pioneering study in which patients have received intraoperative RT instead of a postoperative boost after neoadjuvant therapy with very good outcome results [6].

Therefore, we also welcome larger trials to integrate intraoperative therapy after neoadjuvant therapy in patients with a good response, taking into consideration that the number of pathological complete responses achieved today is significantly higher than in the 1990s, reaching now $40-60 \%$ or more in patients with triple-negative and HER2-overexpressing breast cancer $[6,7]$.

In summary, with the rapid progress in systemic therapy for early breast cancer and the integration of tumor biology in current treatment concepts, it has become increasingly difficult to integrate trial data from 10 or 20 years ago into current 
breast cancer management. This obviously impacts particularly on medical specialities where long-term follow-up is necessary before definite conclusions can be made. Therefore, in line with the data of the UK START trials on hypofractionated breast irradiation with 10-year follow-up of 2 randomized controlled trials and the comment from highly experienced international colleagues from radiation oncology recently published in Lancet Oncology, we welcome the integration of tumor biology in the multidisciplinary concepts for our breast cancer patients. We also welcome intelligent new approaches like intraoperative RT. The authors of this Consensus are already working very closely with radiation oncologists at their centers and we are all very motivated to participate in innovative trials together with our colleagues from radiation oncology.

For the authors: Michael Untch, Berlin

\section{References}

1 Goldhirsch A, Wiener EP, Coates AS, et al.: Personalizing the treatment of women with early breast cancer: Highlights of the St. Gallen International Expert Consensus on the Primary Therapy of Early Breast canCer. Ann Oncol 2013;24:22062223.

2 Haviland SJ, Owen JR, Dewar JA, et al., on behalf of the START Trialist' Group: The UK Standardisation of Breast Radiotherapy (START) trials of radiotherapy hypofractionation for treatment of early breast cancer: 10-year follow-up result of two randomised controlled trials. Lancet Oncol 2013; 14:1086-1094.
3 Haffty BG, Buchholz TA: Hypofractionated breast radiation: Preferred standard of care? Lancet Oncol 2013;14:1032-1034.

4 Giuliano AE, Hunt KK, Ballman KV, et al.: Axillary dissection versus no axillary dissection in women with invasive breast cancer and sentinel node metastasis. JAMA 2011;305:569-575.

5 AGO Guidelines www.ago-online.de.

6 Fastner G, Reitsamer R, Menzel C, et al.: IOERT und Ganzbrustbestrahlung nach neoadjuvanter Chemotherapie beim brusterhaltend operierten Mammakarzinom. Strahlenther Onkol 2013;189: 176-1.
7 Untch M, Rezai M, Loibl S, et al.: Neoadjuvant treatment with trastuzumab in HER2-positive breast cancer: Results from the GeparQuattro study. J Clin Oncol 2010; 28:2024-2031.

8 von Minckwitz G, Eidtmann P, Rezai M, et al.: Neoadjuvant chemotherapy and bevacizumab for HER2-negative breast cancer. N Engl J Med 2012;26:299-309. 